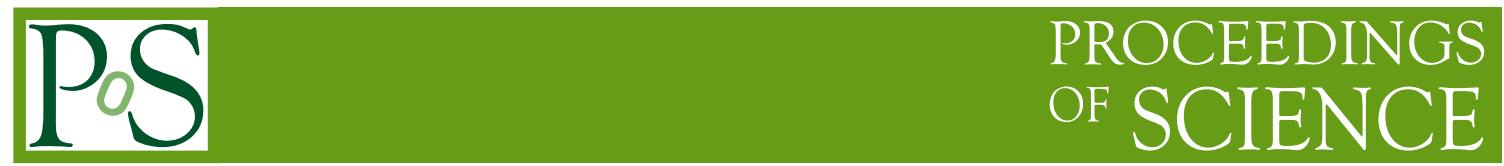

\title{
The Triple-GEM Project for the Phase 2 Upgrade of the CMS Muon System
}

D. Abbaneo ${ }^{15}$, M. Abbas ${ }^{15}$, M. Abbrescia ${ }^{2}$, A.A. Abdelalim ${ }^{8}$, M. Abi Akl ${ }^{13}$, W. Ahmed ${ }^{8}$, W. Ahmed ${ }^{17}$, P. Altieri ${ }^{2}$, R. Aly ${ }^{8}$, A. Ashfaq ${ }^{17}$, P. Aspell ${ }^{15}$, Y. Assran ${ }^{7}$, I. $A w a n^{17}$, S. Bally ${ }^{15}$, Y. Ban ${ }^{3}$, S. Banerjee ${ }^{19}$, P. Barria ${ }^{5}$, L. Benussi ${ }^{14}$, V. Bhopatkar ${ }^{22}$, S. Bianco ${ }^{14}$, J. Bos ${ }^{15}$, O. Bouhali ${ }^{13}$, S. Braibant ${ }^{4}$, S. Buontempo ${ }^{24}$, J. Cai ${ }^{3}$, C. Calabria ${ }^{2}$, C. Caputo $^{2}$, F. Cassese ${ }^{24}$, A. Castaneda ${ }^{13}$, S. Cauwenbergh ${ }^{16}$, F.R. Cavallo ${ }^{4}$, A. Celik ${ }^{9}$, M. Choi ${ }^{31}$, K. Choi ${ }^{31}$, S. Choi ${ }^{29}$, J. Christiansen ${ }^{15}$, A. Cimmino ${ }^{16}$, S. Colafranceschi ${ }^{15}$, A. Colaleo ${ }^{2}$, A. Conde Garcia ${ }^{15}$, M.M. Dabrowski ${ }^{15}$, G. De Lentdecker ${ }^{5}$, R. De Oliveira ${ }^{15}$, G. de Robertis ${ }^{2}$, S. Dildick $^{9,16}$, B. Dorney ${ }^{15}$, W. Elmetenawee ${ }^{8}$, G. Fabrice ${ }^{27}$, S. Ferry ${ }^{15}$, P. Giacomelli ${ }^{4}$, J. Gilmore ${ }^{9}$, L. Guiducci ${ }^{4}$, A. Gutierrez ${ }^{12}$, R.M. Hadjiiska ${ }^{28}$, A. Hassan ${ }^{8}$, J. Hauser ${ }^{21}$, K. Hoepfner ${ }^{1}$, M. Hohlmann ${ }^{22}$, H. Hoorani ${ }^{17}$, Y.G. Jeng ${ }^{18}$, T. Kamon ${ }^{9}$, P.E. Karchin ${ }^{12}$, H. Kim ${ }^{18}$, S. Krutelyov ${ }^{9}$, A. Kumar ${ }^{11}$, J. Lee ${ }^{31}$, J. Lee $^{31}$, T. Lenzi ${ }^{5}$, L. Litov ${ }^{28}$, F. Loddo ${ }^{2}$, T. Maerschalk ${ }^{5}$, G. Magazzu ${ }^{26}$, M. Maggi ${ }^{2}$, Y. Maghrbi ${ }^{13}$, A. Magnani ${ }^{25}$, N. Majumdar ${ }^{19}$, P.K. Mal ${ }^{6}$, K. Mandal ${ }^{6}$, A. Marchioro' ${ }^{15}$, A. Marinov ${ }^{15}$, J.A. Merlin ${ }^{15}$, N. Mohammed ${ }^{11}$, A.K. Mohanty ${ }^{23}$, A. Mohapatra ${ }^{22}$, S. Muhammad ${ }^{17}$,

S. Mukhopadhyay ${ }^{19}$, S. Nuzzo ${ }^{2}$, E. Oliveri ${ }^{15}$, L.M. Pant ${ }^{23}$, P. Paolucci ${ }^{24}$, I. Park ${ }^{31}$, G. Passeggio ${ }^{24}$, B. Pavlov ${ }^{28}$, B. Philipps ${ }^{1}$, M. Phipps ${ }^{22}$, D. Piccolo ${ }^{14}$, H. Postema ${ }^{15}$, G. Pugliese ${ }^{2}$, A. Puig Baranac ${ }^{15}$, A. Radi ${ }^{7}$, R. Radogna ${ }^{2}$, G. Raffone ${ }^{14}$, S. Ramkrishna ${ }^{11}$, A. Ranieri ${ }^{2}$, C. Riccardi ${ }^{25}$, A. Rodrigues ${ }^{15}$, L. Ropelewski ${ }^{15}$, S. Roychoddhury ${ }^{19}$, M.S. Ryu ${ }^{18}$, G. Ryu ${ }^{31}$, A. Safonov ${ }^{9}$, A. Sakharov ${ }^{10}$, S. Salva ${ }^{16}$, G. Saviano ${ }^{14}$, A. Sharma ${ }^{15}$, S.K. Swain ${ }^{6}$, J.P. Talvitie ${ }^{15,20}$, C. Tamma ${ }^{2}$, A. Tatarinov ${ }^{9}$, N. Turini ${ }^{26}$, T. Tuuva ${ }^{20}$, J. Twigger ${ }^{22}$, M. Tytgat ${ }^{* 16}$, I. Vai ${ }^{25}$, M. van Stenis ${ }^{15}$, R. Venditi ${ }^{2}$, E. Verhagen ${ }^{5}$, P. Verwilligen ${ }^{2}$, P. Vitulo ${ }^{25}$, U. Yang ${ }^{30}$, Y. Yang $^{5}$, R. Yonamine ${ }^{5}$, N. Zaganidis $^{16}$, F. Zenoni ${ }^{5}$ 


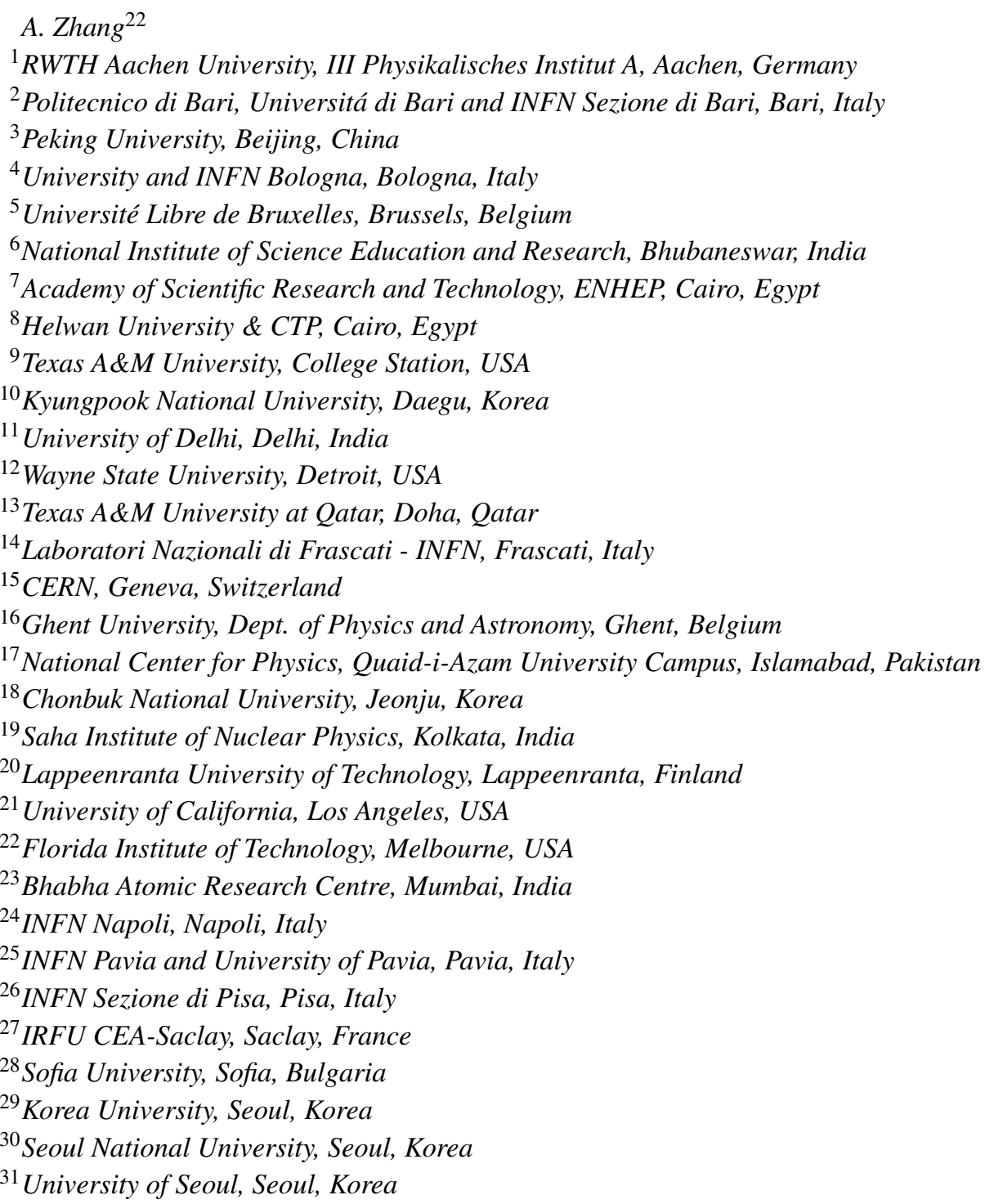

\section{E-mail: Michael. Tytgat@UGent.be}

In view of the high-luminosity phase of the LHC, the CMS Collaboration is considering the use of Gas Electron Multiplier (GEM) detector technology for the upgrade of its muon system in the forward region. With their ability to handle the extreme particle rates expected in that area, such micro-pattern gas detectors can sustain a high performance and redundant muon trigger system. At the same time, with their excellent spatial resolution, they can improve the muon track reconstruction and identification capabilities of the forward detector, effectively combining tracking and triggering functions in one single device. The present status of the CMS GEM project will be reviewed, highlighting importants steps and achievements since the start of the R\&D activities in 2009. The baseline design of the triple-GEM detectors proposed for installation in different stations of the CMS muon endcap system will be described, along with the associated frontend electronics and data-acquisition system. The expected impact on the performance of the CMS muon system will be discussed, and results from detector tests, both in the lab and in test beams will be presented. 


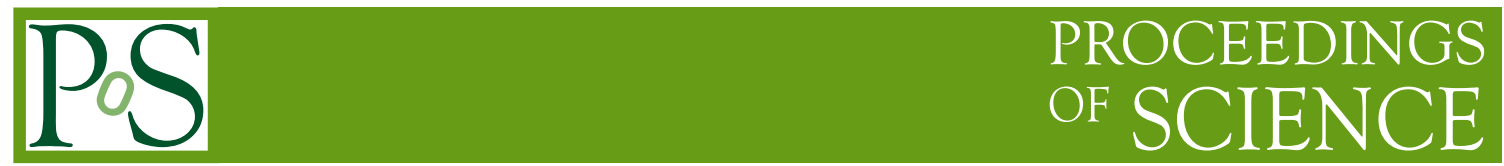

Technology and Instrumentation in Particle Physics 2014

2-6 June, 2014

Amsterdam, the Netherlands

* Speaker. 


\section{Introduction}

Muon detection and reconstruction plays an important role in the physics explorations performed by the CMS Collaboration [1] at the CERN Large Hadron Collider (LHC). The presence of isolated high- $p_{T}$ muons in the final state of $\mathrm{p}-\mathrm{p}$ collisions may signal potentially interesting events. A few typical examples where a large muon detection coverage and efficiency is essential are e.g. Higgs boson decays into four muons, leptonic decays of $\mathrm{W}$ bosons and top quarks and decay chains of supersymmetric particles in which small mass splittings occur. Ensuring a highly performing muon system over the years is therefore of utmost importance.

In view of the LHC Phase 2 era, or High Luminosity LHC (HL-LHC) starting around 2023, where p-p collisions are foreseen at $14 \mathrm{TeV}$ with peak luminosities of $5-10 \times 10^{34} \mathrm{~cm}^{-2} \mathrm{~s}^{-1}, \mathrm{CMS}$ is planning to upgrade several subsystems [2], including its muon system. As shown in Fig. 1, the present CMS muon system consists of three complementary types of gaseous detectors [3]: Drift Tubes (DT) and Cathode Strip Chambers (CSC) that are used for precise tracking and triggering, and Resistive Plate Chambers (RPC) that provide an additional muon trigger.

In the largest part of the muon system, a combination of two subsystems is used, with DT and RPC in the barrel part, and CSC and RPC in the endcaps. However, the $1.6<|\eta|<2.4$ region of the endcaps is equipped with CSC only, leaving this part less robust, with low redundancy, while it has a relatively high particle track density. In addition, the muon momentum determination from particle bending in the CMS magnetic field, is hampered in this particular region as the lever arm to measure the bending angle in each station is short with CSC only. As a result, many low- $p_{T}$ muons that undergo multiple scattering in the muon system and the iron return yoke are misidentified as high- $p_{T}$ tracks, leading to a flattening of the muon trigger rate towards high momenta. In order to maintain a highly efficient muon trigger with a low $p_{T}$ threshold at high luminosity, additional detector layers are required in this area, where high spatial resolution is particularly needed in the inner endcap stations where the bending of the muons is largest.

Next to the muon system, HL-LHC upgrades are also foreseen for the CMS tracker and calorimeter. The inner silicon tracker coverage will be extended into the $|\eta|>2.4$ region. In addition, a Track Trigger will be implemented providing charged particle candidates in the inner tracker that can be matched to muon candidates in the Level-1 Trigger. In order to also extend the coverage of the muon system in the $|\eta|>2.4$ region, one can profit from the foreseen rebuild of the endcap calorimeter to add muon detection layers in front of the present first muon endcap station.

\section{The CMS GEM project}

The original design of CMS actually included RPC detectors for the $1.6<|\eta|<2.4$ region of the endcaps. However, the standard CMS RPC cannot handle the hit rates of a few $\mathrm{kHz} / \mathrm{cm}^{2}$ that are expected in that region for the HL-LHC. Instead, the use of Gas Electron Multipliers (GEM) [4] is being proposed to instrument part of the muon endcap system. GEM in addition to high rate capability $\left(\sim \mathrm{MHz} / \mathrm{cm}^{2}\right)$ offer high longevity, high spatial resolution $(\sim 100 \mu \mathrm{m})$ suitable for tracking and sufficient time resolution $(\sim \mathrm{ns})$ for triggering purposes. At present, a scenario with 3 new stations equipped with GEM is proposed as indicated in Fig. 1: the first two muon endcaps, where the muon momentum determination is most effective, are to be equipped in the 


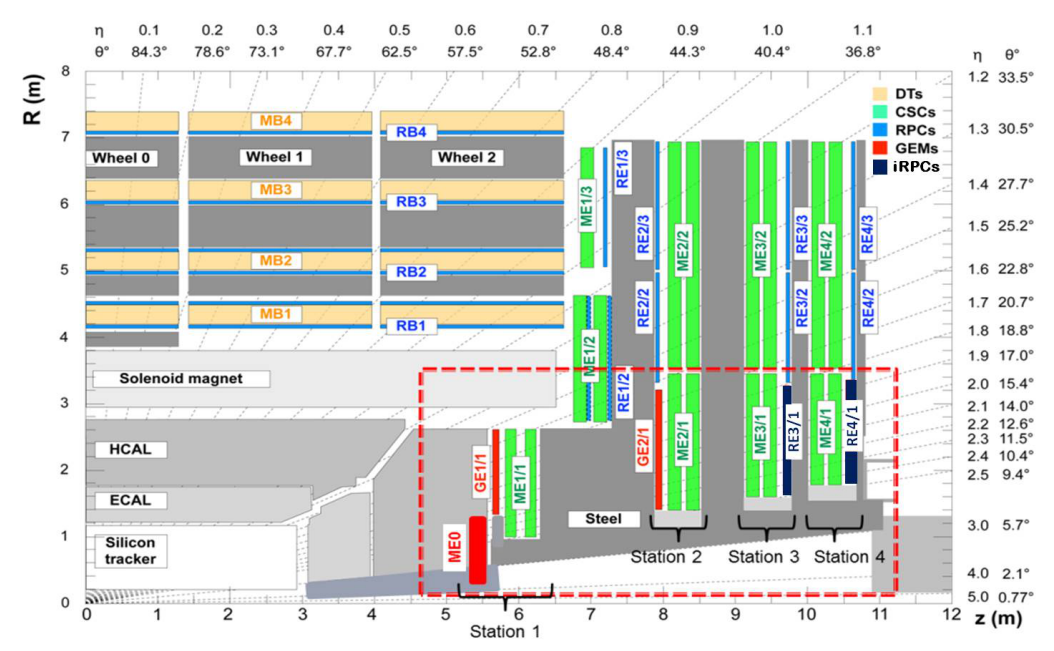

Figure 1: A quadrant of the CMS muon system, showing the different subsystems. The locations of the new forward detectors proposed for Phase 2 are contained in the dashed box; the ME0, GE1/1 and GE2/1 GEM stations are indicated in red.
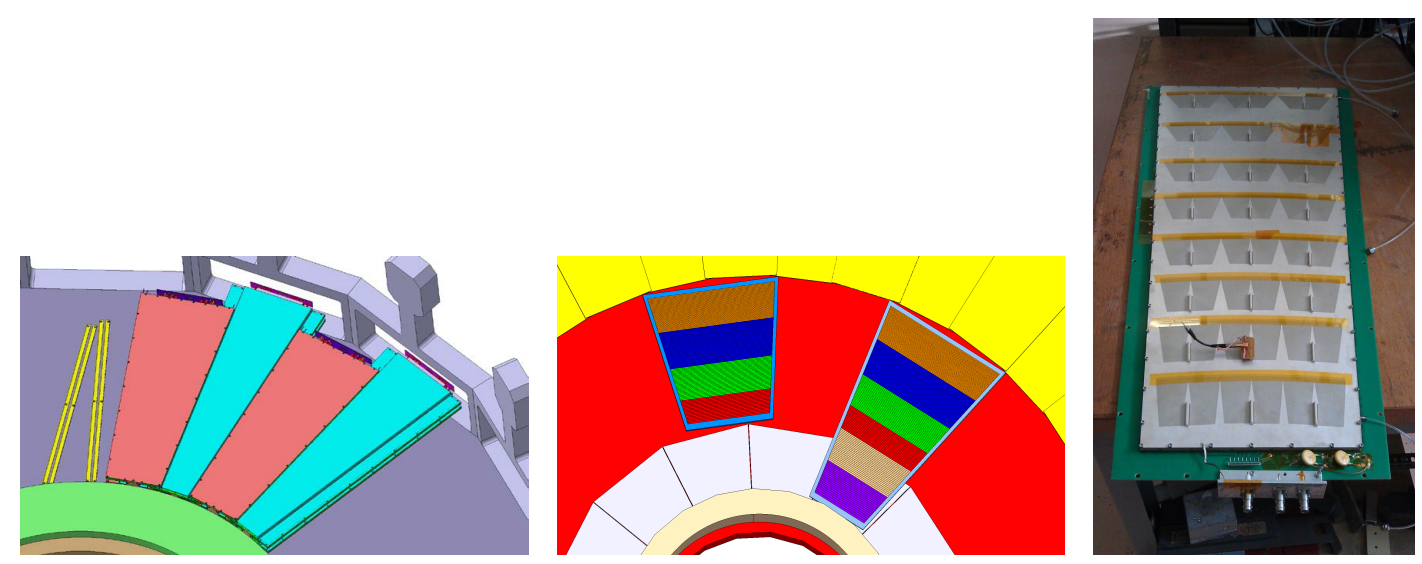

Figure 2: Layout of the GE1/1 (left) and GE2/1 (middle) stations and photograph of a basic GE1/1 detector unit (right).

$1.5<|\eta|<2.4$ region with double layers of GEM, denoted as GE1/1 and GE2/1; behind the new, more compact endcap calorimeter proposed for the HL-LHC, an additional station of 6 GEM layers denoted as ME0, is to be installed to extend the muon system into the $|\eta|>2.4$ region. The maximum hit rate expected for ME0 is $100 \mathrm{kHz} / \mathrm{cm}^{2}$ during the HL-LHC, which is still sustainable by GEM. For the third and fourth endcaps, additional layers of improved RPC (iRPC) with higher rate capability compared to the standard CMS RPC are foreseen, denoted as RE3/1 and RE4/1, to improve the muon system redundancy even more.

The general layout of the GE1/1 and GE2/1 stations is depicted in Fig. 2. The basic, trapezoidalshaped chamber units contain a triple-GEM detector. Super-chambers are formed by stacking together two basic units, yielding two readout points spaced $20 \mathrm{~mm}$ apart, with a total detector thickness of $88 \mathrm{~mm}$. For GE1/1, 36 super-chambers, each covering $10^{\circ}$ in $\phi$, will be installed in each endcap, giving a total of 144 basic chambers units to be produced. To maximize the coverage 

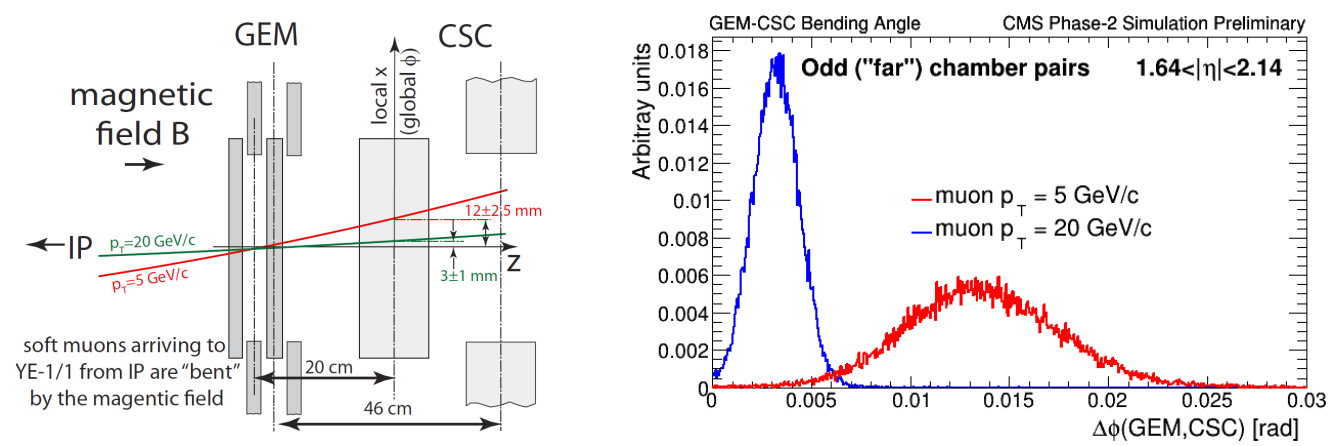

Figure 3: Left: Illustration of the principle of combining the GEM and CSC layers in each station to enlarge the lever arm for the bending angle measurements. Right: Simulation of the bending angle measurement in the first endcap station (GE1/1-ME1/1) for soft $(\sim 5 \mathrm{GeV})$ and hard $(\sim 20 \mathrm{GeV})$ muons.

of the detectors in the space available in the YE1 nose, the superchambers will overlap in $\phi$ and alternate between long $(1.5<|\eta|<2.2)$ and short $(1.6<|\eta|<2.2)$ versions taking the support structures into account. For GE2/1, $20^{\circ}$ super-chambers would be used to cover the $1.5<|\eta|<2.4$ region. Due to the neutron shielding already present, again a long and short chamber version will be used. For ME0, a 6 layer system with $20^{\circ}$ chambers is foreseen in the $2.0<|\eta|<\sim 3.5$ region, to be installed in about $30 \mathrm{~cm}$ of space behind the upgraded endcap calorimeter. The final design, including additional neutron shielding, is still under study.

\section{Expected impact on muon trigger performance}

With the additional GEM layers in the endcaps, the lever arm to determine the muon bending angle can be enlarged by combining the data from the GEM and CSC stations, as depicted in Fig. 3. As an example, a simulation of the bending angle measurement in the first endcap (GE1/1-ME1/1 combination) is shown to provide a clear separation between soft and hard muons, which can be used to reduce the muon trigger rate significantly. A simulation of the effect of additional GEM layers on the muon trigger rate expected for HL-LHC conditions is shown in Fig. 4.

\section{Basic detector design and construction}

The GEM foils for the detector prototypes have so far all been produced at the CERN Surface Treatment Workshop using the photolitographic hole etching procedure. The recently introduced single-mask technique [5] is used for the production of the foils for the large-area detectors. For the assembly of the triple-GEM detectors themselves, a novel, cost effective NS2 (no-spacer, nostretch) technique has been developed permitting the chambers to be assembled without the use of any glue nor spacers in the active detector volume [6]. With NS2 technology, one full-scale GE1/1 chamber can be assembled in about two hours only, which is to be compared to the one week that was needed for an assembly using the former glueing technique.

The baseline detector is the GE1/1 chamber shown in Fig. 2. The triple-GEM has a trapezoidalshaped active area of $990 \times(220-455) \mathrm{mm}^{2}$ with a $3 / 1 / 2 / 1 \mathrm{~mm}$ drift/transfer- $1 /$ transfer-2/induction field gap configuration. The bottom of the assembly is the Drift Board, which is a printed circuit 

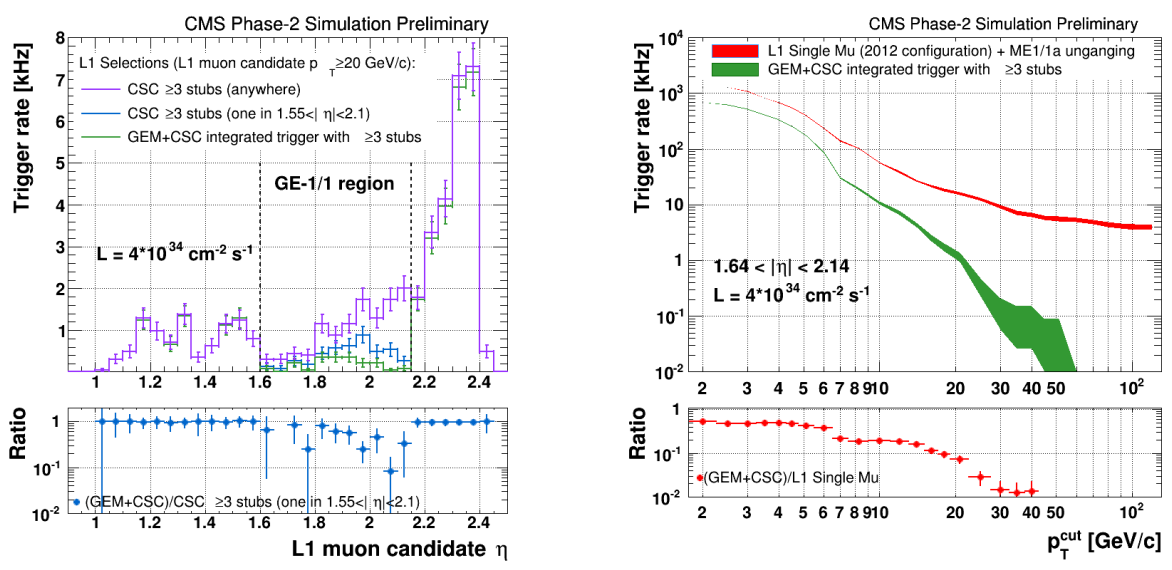

Figure 4: Simulation of the inclusive muon trigger rate expected for the LHC Phase 2 as a function of $\eta$ (left) and of the Level-1 $p_{T}$ trigger threshold for the $1.6<|\eta|<2.1$ region (right). In the latter figure the situation after adding GEM layers is compared with the 2012 muon configuration where in addition the removal ("unganging") of the present 3:1 ganging of channels in the high- $\eta$ half of the ME1/1 CSC station is taken into account, as foreseen in the upgrade. The effect of adding the GE1/1 station is clearly visible.

board holding the drift electrode and the GEM voltage divider. The top of the assembly is the Readout Board containing on the inner side a pattern of readout strips, radially oriented readout along the long side of the chamber. The readout is segmented in up to $10 \times 3(\eta, \phi)$ partitions with 128 strips each, with a strip pitch ranging between $0.6-1.2 \mathrm{~mm}$. The GE2/1 chambers will be similar to the GE1/1 basic unit, albeit with dimensions of about $1.2 \times 0.8 \mathrm{~m}^{2}$ requiring some further R\&D to produce detectors of this size. The ME0 chamber layout is still under study. In case the installation of the full system is approved by CMS, the detector assembly and quality control will be shared by several institutes. A total of $10 \mathrm{GE} 1 / 1$ prototypes have already been built at candidate production sites at CERN, Floride Institute of Technology, Ghent University and INFN Frascati and Bari.

\section{Front-end electronics and data-acquisition}

The GEM readout system, where for the design the GE1/1 station is taken as baseline, should be able to provide fast logical OR trigger information, with a granularity of 2 channels, to be combined locally with the CSC data in the trigger system. At the same time, for every triggered event in CMS, full granularity information is required for the muon reconstruction. A diagram of the main components of the proposed readout system for a single chamber is shown in Fig. 5. The system is divided in two parts: the on-detector part which is on or close to the chamber, and the off-detector part which is to be installed in the CMS counting room.

Electrical cables provide each chamber with high and low voltage. DC/DC regulators mounted on the chamber provide the correct voltage levels to the on-detector electronics. Every GEM detector is subdivided in sectors in both the $\phi$ and $\eta$ direction, with every sector containing 128 readout strips on which charge is induced by the passage of an ionizing particle through the detector volume. All strips of a single sector are connected to one front-end ASIC, through a 128-channel panasonic connector mounted on the outside of the GEM Readout Board. The front-end ASICs are mounted on hybrids that plug into the connectors on the Readout Board. The control, read- 


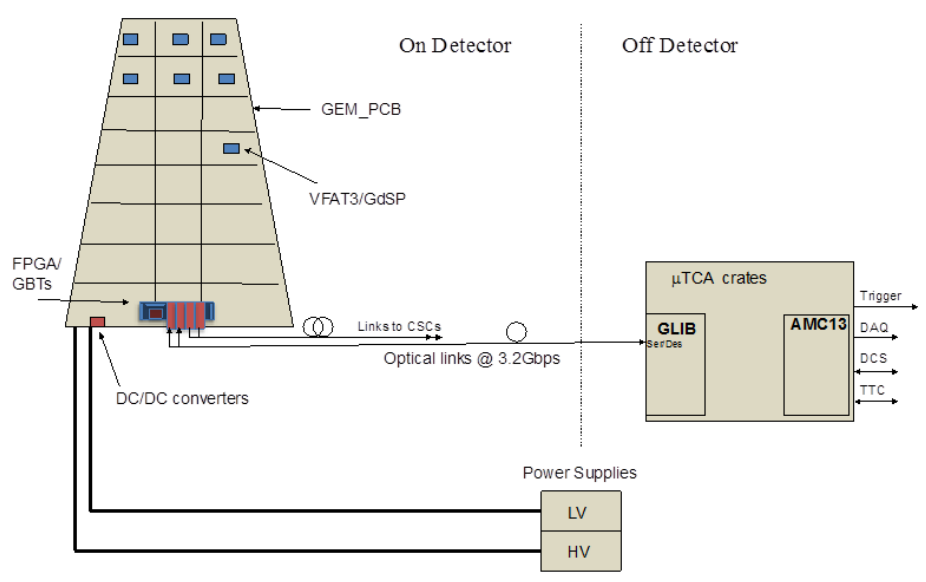

Figure 5: Diagram of the proposed GEM electronics readout system.

out and power to/from these hybrids is delivered via electrical signals (E-links) running through a large printed circuit board called the GEM Electronic Board (GEB) covering the entire detector surface. The communication to the off-detector electronics is performed through optical links via a mezzanine board, called Opto-hybrid, that also plugs into the GEB and contains an FPGA, Gigabit-Transceiver (GBT) chipsets [7], and 2 optical connectors. The off-detector electronics are implemented in the $\mu \mathrm{TCA}$ standard and provide the interface to the CMS trigger, data-acquisition and control systems. There are two optical paths to/from the Opto-hybrid. The first one is bidirectional and runs between the $\mu \mathrm{TCA}$ crates in the counting room and the Opto-hybrid. This path is used for sending setup and control signals to the front-end chips. The return path is used for full-granularity tracking data and returning slow control data. The second path is uni-directional and takes fixed-latency trigger data from the GEM system to the CSC Trigger Mother Board for the Level-1 muon trigger.

For the GE1/1 station, the VFAT3 front-end ASIC was chosen, which is still under development as the successor of the VFAT2 chip [8]. It contains a 128-channel charge sensitive preamplifier, shaper and constant fraction discriminator producing digital output. The data is split into two paths, one with a fixed latency for trigger purposes, and one for tracking data with variable latency. The VFAT3 has a programmable shaping time and gain in order to optimize the charge collection from the GEM detector while keeping an excellent timing resolution; it is designed for high rates, and interfaces directly to the GBT chipset.

\section{Detector performance}

The performance of the CMS triple-GEMs has been evaluated both in the RD51 lab at CERN with X-ray sources and in many test beam campaigns with pion/muon beams at the CERN SPS [10] and more recently at FNAL. The chambers were operated mainly with a $\mathrm{Ar}_{\mathrm{CO}_{2}}: \mathrm{CF}_{4}$ 45:15:40 gas mixture. The readout was done using either digital TURBO/VFAT2-based front-end electronics [8] or the Scalable Readout System (SRS) [9] with an analog APV25 hybrid front-end.

It was demonstrated that the detectors exhibit a broad efficiency plateau with maximum efficiency above $98 \%$. A time resolution of 4 ns was obtained during drift and induction field scans. 

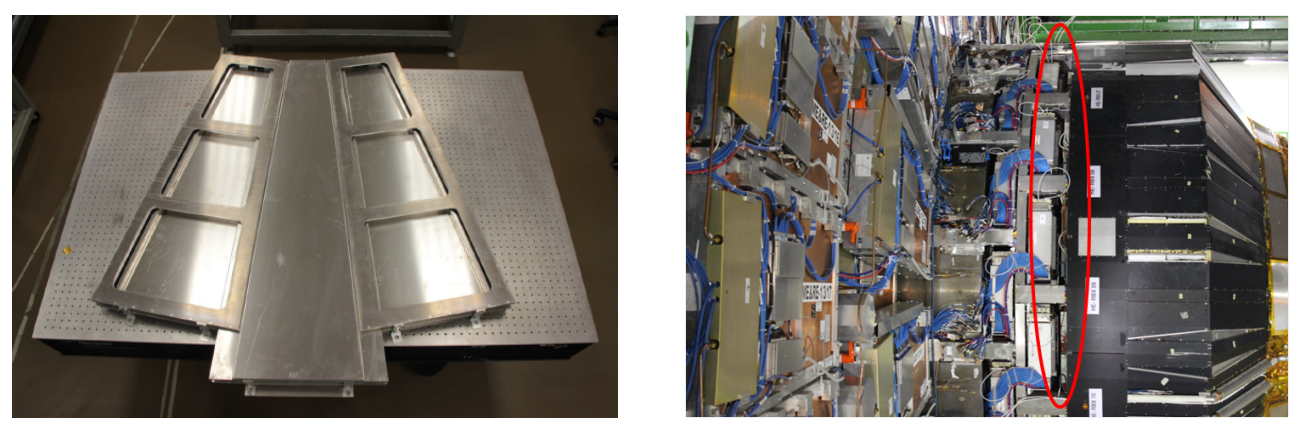

Figure 6: Left: Photograph of the 2nd set of GE1/1 super-chamber dummies used for trial insertion into one of the inner CMS endcap stations. Right: Photograph of the actual situation in the nose of one of the first endcap stations; the location foreseen for the GE1/1 super-chambers is indicated by the red oval shape.

Spatial resolutions of the order of $270 \mu \mathrm{m}$ were measured with a digital readout, compatible with what one expects from the strip pitch; using analog readout, spatial resolutions below $110 \mu \mathrm{m}$ were found. It was shown that the detector performance was not affected significantly in the presence of an external magnetic field similar to the one expected inside CMS. Gain measurements performed using an X-ray gun on a GE1/1 detector showed that the variation in gain remained below $15 \%$ across the detector surface [6]. Finally, ongoing detector irradiation tests with a ${ }^{137} \mathrm{Cs}$ source at the CERN GIF facility revealed no aging effects so far, after an accumulated dose of $7 \mathrm{mC} / \mathrm{cm}^{2}$ [11].

\section{Integration into CMS}

Anticipating a possible installation of the full GE1/1 station in the 2nd LHC Long Shutdown (2018-2019), most of the services and cabling needed for this station is being put in place in the CMS experimental area already now during the ongoing 1st LHC Long Shutdown (2013-2015).

To finalize the super-chamber layout, two sets of GE1/1 super-chamber dummies were produced: a first set with preliminary chambers dimensions, and a second set shown in Fig. 6, produced according to the final, alternating long-short chamber geometry. The dummies did not contain any detectors, nor any electronics, however, the weight was similar to the real super-chambers. As can be seen in the figure, the space available for the GE1/1 super-chambers inside CMS is quite limited (about $100 \mathrm{~mm}$ ). Moreover, the forward GE1/1 region is densely packed in terms of detector cabling and services. Apart from verifying the dimensions of the chambers and the detector insertion procedure itself, also the positions of the connections for gas, cooling, electronics and detector powering need to be optimized. A first trial installation of the first set of dummies into one of the inner endcaps was succesfully performed during the Summer of 2013; a second trial installation with the second set was done in March this year. During the chamber insertion, rails were used with T-grooves to slide and rotate the chambers into their final position.

\section{Summary and outlook}

In the framework of the RD51 Collaboration, the CMS GEM project was initiated in 2009 to provide a feasible solution for the CMS muon system upgrade towards the HL-LHC. After 5 years of R\&D, the design and characterization of the CMS triple-GEM chambers is now close to final. 
The present scope of the project proposes the installation of 3 new stations: the GE1/1 and GE2/1 stations in the $1.5<|\eta|<2.4$ region of the first and second endcap disks respectively will add redundancy to the system and will improve its trigger and tracking capabilities; the ME0 station at the back of the rebuild endcap calorimeter will extend the reach of the muon system to $|\eta| \approx 3$ or more, matching the corresponding upgrade of the pixel tracker.

Preparations for the installation of a demonstrator system during the 2016 Year-End Technical Stop are ongoing. This system will consist of 2 GE1/1 super-chambers covering a $20^{\circ}$ sector of one inner endcap disk at $1.5<|\eta|<2$.2. It will allow to perform a complete system validation in-situ, to gain operational experience with GEMs inside the CMS detector, and to test the integration of the GEM and CSC system at trigger level. The installation of the full GE1/1 station could then be done as early as the 2nd LHC Long Shutdown, followed by the installation of GE2/1 and ME0 during the 3rd LHC Long Shutdown near 2022.

\section{Acknowledgments}

We acknowledge the continuous technical support from the RD51 Collabortion for this project. The corresponding author would like to thank the FWO-Flanders (Belgium) for the funding received for this work.

\section{References}

[1] CMS Collaboration, The CMS experiment at the CERN LHC, J. Instrum. 3 (2008) S08004

[2] CMS Collaboration, Technical proposal for the upgrade of the CMS detector through 2020, CERN-LHCC-2011-006, CMS-UG-TP-1; CMS Collaboration, Technical proposal for the Phase 2 upgrade of the CMS detector, in preparation

[3] CMS Collaboration, The CMS muon project: Technical Design Report, CERN-LHCC-97-32, CMS-TDR-003

[4] F. Sauli, GEM: A new concept for electron amplification in gas detectors, Nucl. Instrum. Meth. A 386 (1997) 531

[5] M. Villa et al., Progress on large area GEMs, Nucl. Instrum. Meth. A 628 (2011) 182

[6] D. Abbaneo et al., Status of the Triple-GEM Project for the Upgrade of the CMS Muon System, J. Instrum. 8 (2013) C12031

[7] P. Vichoudis et al., The Gigabit Link Interface Board (GLIB), a flexible system for the evaluation and use of GBT-based optical links, J. Instrum. 5 (2010) C110167

[8] P. Aspell et al., The VFAT production test platform for the TOTEM experiment, Proc. of the Topical Workshop on Electronics for Particle Physics 2008, CERN-2008-008

[9] S. Martoiu et al., Front-end electronics for the Scalable Readout System of RD51, 2011 IEEE Nucl. Sci. Symp. Conf. Rec. 2036-2038

[10] D. Abbaneo et al., Beam Test Results for New Full-scale GEM Prototypes for a Future Upgrade of the CMS High- $\eta$ Muon System, 2012 IEEE Nucl. Sci. Symp. Conf. Rec. 1172-1176

[11] D. Abbaneo et al., Study of long-term operation of triple-GEM detectors for the high rate environment in CMS, Proc. of the 3rd International Conference on Micro Pattern Gaseous Detectors, 1-6 July, 2013, Zaragoza, Spain (MPGD2013), J. Instrum., in print 